\title{
Treatment of ADHD in Perinatal Women with Dual Diagnosis: A Comprehensive Pharmacological and Psychosocial approach
}

\author{
Anupriya Gogne ${ }^{1,2 *}$ and Rosemary M Ryan ${ }^{3}$ \\ ${ }^{1}$ Department of Medicine, Women \& Infants Hospital of Rhode Island, Providence, RI, USA \\ ${ }^{2}$ Department of Psychiatry and Human Behavior, Alpert Medical School of Brown University, Providence, RI, USA \\ ${ }^{3}$ Center for Women's Behavioral Health, Women \& Infants Hospital of Rhode Island, Providence, RI, USA
}

Submission: March 11, 2021; Published: March 19, 2021

*Corresponding author: Anupriya Gogne MD, Attending Psychiatrist, Center for Women's Behavioral Health Women \& Infants Hospital, USA

Abstract

Attention deficit hyperactivity disorder (ADHD) is a chronic neurodevelopmental disorder affecting about 4.4 percent of adults in the United States, with females presenting more commonly with inattention rather than impulsivity and hyperactivity. The perinatal period, followed by transition to motherhood, presents greater challenges for women with ADHD as there is significant worsening of pre-existing deficits in executive functioning due to major hormonal changes, prolonged sleep deprivation, heightened social demands and abrupt discontinuation of stimulants. In this report, we discuss the case of M, a middle-aged woman suffering from inadequately treated ADHD, comorbid with Bipolar disorder, TBI (Traumatic Brain Injury) and history of substance use. We describe the patient's clinical presentation during pregnancy, difficulties in engagement, and a treatment course with multiple complications. More specifically, we highlight our unique implementation of a long-term treatment approach to address her ADHD-related challenges during the postpartum period and subsequent years as M cares for her children with limited finances and social supports during a global pandemic. Finally, we emphasize outcomes of treatment and future directions for clinical research to design outpatient treatment models individualized to fit the complex needs of this population.

Keywords: ADHD; Perinatal; Dual diagnosis; Substance use; Psychosocial interventions

Abbreviations: ADHD: Attention Deficit Hyperactivity Disorder; TBI: Traumatic Brain Injury

\section{Summary/Background}

Attention deficit hyperactivity disorder (ADHD) is a neurodevelopmental disorder that can continue to affect mental health and daily functioning into adulthood. In the United States, estimated prevalence of adult ADHD is 4.4 percent [1]. Compared to males with ADHD, females with ADHD are more vulnerable to inattention than hyperactivity and impulsivity. Also, females are diagnosed with ADHD significantly later than males [2]. In 2012, a longitudinal study showed that a majority of girls experience ADHD symptoms and impaired functioning more than a decade later [3]. Of note, several studies confirm comorbidity of ADHD with affective disorders, as well as substance use [4].

The perinatal period, followed by the transition to motherhood, presents greater challenges for women with ADHD. Risk factors for worsening of pre-existing ADHD include prolonged sleep deprivation [5], and major changes in external environment [6]. Understandably, caregiving creates increased demands in executive functioning. Post-partum hormonal changes, and abrupt discontinuation of medications due to concern about safety of stimulants during pregnancy and lactation [7], further worsen ADHD symptoms. It is reasonable to foresee that these challenges can extend into subsequent motherhood as the child starts school, especially if the child also develops ADHD. These symptoms interfere with daily functioning and, in turn, create a vicious cycle of worsening distress and relapse to harmful substances, particularly if these substances were used to selfmedicate untreated symptoms in the past. 


\section{Introduction}

We present the case of $\mathrm{M}$, a middle-aged woman suffering from inadequately treated ADHD, comorbid with Bipolar disorder, TBI (Traumatic Brain Injury), and history of polysubstance use. We describe the patient's clinical presentation during pregnancy, difficulties in engagement, and a treatment course with multiple complications such as severe manic psychosis with relapse, multiple head injuries, and involvement with legal and child welfare agencies. We discuss her ADHD-related challenges persisting into the first two years postpartum, despite stabilization of bipolarity and sobriety. We highlight the challenges ADHD presents for mothers with multiple children during a global pandemic, with financial stressors and limited social supports adding to that stress. Finally, we discuss outcomes of this longterm, individualized treatment.

Treating such complex psychopathology can be overwhelming for most clinicians. It can be difficult to decide which clinical symptoms to address first. Moreover, poor treatment engagement inherent in attachment and substance use disorders complicates matters. Our goal is to describe a collaborative, comprehensive long-term treatment model for patients with complex needs. We describe how multi-disciplinary clinicians can implement pharmacological and psychotherapeutic interventions at different levels of care to treat distressing ADHD symptoms and maintain sobriety.

This patient provided informed consent for the publication of this report.

\section{Presenting Complaints}

M is a 43-year-old, G4P3, single, Caucasian woman presenting to our outpatient clinic at nine weeks gestation. She was notably anxious, fidgety, unable to sit still, talking fast and easily distracted. She displayed insight into her symptoms from prior diagnosis of Bipolar Disorder, Type 1 with psychotic features. She was aware that she was in the midst of a hypomanic episode, with decreased need for sleep, racing thoughts, euphoria, grandiosity and impulsivity. She had no psychotic symptoms at the time but reported a history of psychotic episodes without medications (Lithium, antipsychotics). She expressed concern about her risk-taking behaviors during such episodes. Although she could not fully recall her actions, other people had informed her that she engaged in impulsive sexual encounters (one of her older children was conceived during a manic episode), illegal acts (stole a car), and used substances, eventually resulting in psychiatric hospitalization.

M lost custody of her two older children due to multiple similar decompensations secondary to poor treatment adherence and ongoing substance use. She also reported chronic ADHD, first diagnosed after neuropsychological testing as a young adult, responding well to mixed amphetamine salts. Prior to receiving the correct diagnosis and treatment, she reported using cocaine to "self-medicate" as it helped her attention and anxiety.

M had a history of medical comorbidities including seizures (it is unclear whether these were a result of substance withdrawal or intoxication, or an independent seizure disorder), blood pressure fluctuations, and untreated Hepatitis C contracted through intravenous drug use.

\section{Treatment Course}

This unexpected, unplanned, but desired pregnancy motivated M to maintain sobriety and seek treatment voluntarily. Her initial goal was to obtain custody of her infant after delivery. Unfortunately, after our first meeting M failed to call or keep her appointments in our clinic, despite multiple outreach attempts. Months later, she resurfaced, having endured bouts of manic psychosis, police arrests, and being hit by a car while under the influence of alcohol and cocaine. The subsequent few months were difficult for $M$, as she dealt with multiple inpatient admissions, medication changes, alcohol withdrawal, cravings for illicit substances and involvement with child welfare agencies.

Following inpatient hospitalization at a psychiatric hospital, M was referred to our Perinatal Partial Hospital Program. While M was in the partial program, our clinicians monitored her affective symptoms closely while adjusting medication doses and helped her to form healthier social attachments. $M$ received individual as well as group therapy for four weeks, providing structure to help with her ADHD symptoms. M continued substance use treatment outside the partial program, including Alcoholics Anonymous meetings, support from her sponsor, and Buprenorphine for treatment of Opioid Use Disorder.

M's residual hypomanic symptoms (high energy, decreased need for sleep, racing thoughts, and impulsivity) were stabilized with Lithium carbonate titrated to a maximum of $1800 \mathrm{mg}$, and Risperidone $4 \mathrm{mg}$ at bedtime. Lithium levels were monitored weekly. Gabapentin was added at $600 \mathrm{mg}$ three times a day to address insomnia, anxiety and alcohol cravings. For each medication, safety data during pregnancy and breastfeeding was reviewed.

Our team provided $M$ continuous support during the postpartum period, visiting her in the hospital after delivery as she was most vulnerable to relapse and psychiatric decompensation during this time. Child services placed her newborn with a relative at delivery, and subsequent separation from the infant was difficult and depressing. Given M's history of poor response to Selective Serotonin Reuptake Inhibitors (SSRI) in prior medication trials, Lamotrigine was added and titrated up to $150 \mathrm{mg}$ daily for treating depression in the context of underlying mood instability. With this medication regimen, $\mathrm{M}$ achieved psychiatric stability. She also increased her social supports, rebuilding relationships with friends and family. 
During the first post-partum year, M worked with child welfare agencies and family drug court working towards re-unification with her children. Despite supervised visits with her baby, M's distress over this separation from her children increased her risk of relapse. Acutely aware of the risks of losing her children permanently, M worked proactively with her substance use counselor to maintain sobriety. During this period, treatment interventions in our clinic focused on attachment. The strong attachment $M$ had with this author (Gogne) expanded to include other members of our multi-disciplinary team. This therapeutic alliance helped $\mathrm{M}$ to feel safe and respected so she could practice healthier behaviors to cope with distress. After 12-16 months of rigorous outpatient treatment, M maintained stability and was reunited with her children. This joyous reunification, however, presented her with new challenges. Cognitive deficits related to untreated ADHD worsened by multiple TBIs and chronic substance use rendered $\mathrm{M}$ unable to carry out basic tasks of daily functioning without support. For instance, although M sought to reinstate her suspended driver's license to be "more independent," inattention on the road caused significant anxiety and frustration. While doing household chores, she was unable to organize herself, maintain attention and finish tasks. She became easily distracted, often by her own racing thoughts. Most distressingly, she despaired over her inability to help her six-year-old son (who also has ADHD) with homework, adding to shame and guilt of being unable to care for her children optimally. These daily difficulties in functioning adversely affected her sense of self as a mother and contributed to her depressive symptoms.

$M$ responded well to amphetamine salts in the past, with no history of prescription drug misuse. However, given her long term struggle with drugs and alcohol and recent decompensation, we decided to first try non-stimulant medications. Unfortunately, $\mathrm{M}$ had a poor response and tolerability to both Bupropion (palpitations) and Atomoxetine (dizziness). M was apprehensive about a stimulant trial; she did not want to jeopardize the progress she had made. Thus, we collaborated with her to design a controlled and structured stimulant trial with adjunctive nonpharmacological interventions. The trial began with $10 \mathrm{mg}$ of extended-release mixed amphetamine salts (this formulation being low risk for dependence). M noticed improvement but felt "too activated" and unable to fall asleep. We then switched to the immediate release formulation $(10 \mathrm{mg})$, to be taken in the morning. Additionally, we helped her to schedule complex tasks within 4-6 hours of taking the medication to optimize her functioning. Later, when $\mathrm{M}$ needed to homeschool her children during the pandemic, we added an afternoon dose of 5-10 mg to help her with remote schooling. The plan included drug holidays over the weekends.

Treatment emphasized that M maintain sobriety from all illicit substances and alcohol and comply with random urine drug screens. M readily agreed to this, reporting that the drug screens helped her "stay on track" with her recovery. She attended her appointments and took medications as prescribed. Her drug screens remained negative for any illicit substances.

M started bi-weekly individual therapy with the author (Ryan), staggered with medication management appointments. Non-pharmacological interventions were structured as ADHD coaching to manage deficits in executive functioning, thus improving her functioning as a mother. Initial sessions focused on validating M's achievements, a strengths-based treatment approach to leverage M's self-identified "superpowers," including perseverance, commitment to her family, high energy and creative problem solving. Using clinical assessment tools for adult ADHD, we identified M's challenges across most areas of daily functioning, including managing time and money; hyperactivity; sensory overload, and social interactions. With this information, the author (Ryan) helped $M$ to set attainable treatment goals and stay focused on one goal at a time. Initial goals included helping her son with homework; consolidating and streamlining her "to-do" list; completing household tasks more efficiently; creating a household budget to ensure bills were paid promptly; and maintaining a stable sleep pattern (especially important as insomnia can worsen ADHD and mood instability). Treatment interventions included use of a discreet phone application for digital to-do lists; setting time limits for household tasks with alarm reminders (e.g., when to leave the house to pick up children from school), as well as emphasis on mindfulness practices, attention to self-care (sleep hygiene, exercise, and nutrition), and communication and self-advocacy skills.

By the end of 6 months, $M$ had made significant progress in meeting her goals, despite the added stressors of the COVID-19 lockdown (during which $\mathrm{M}$ was responsible for the daily care of her own children plus two nieces-a total of five children under age six). During this time, we scheduled weekly tele-visits with $\mathrm{M}$, monitoring her sleep, focusing on emotional regulation, while normalizing the challenges that all parents faced during the pandemic. Remarkably, not only was $\mathrm{M}$ able to manage complex tasks during this period, but she also successfully managed her son's all-day remote learning, with completion of assignments that he needed to advance to the second grade. Prior to this treatment, $\mathrm{M}$ was unable to sit still and read with her son; with treatment she was able to preview his lessons with the teacher, set up his computer, and assist him every day for three months. Ever since, $\mathrm{M}$ has felt more confident and continued to make progress towards establishing an independent household. She consolidated her extensive medical and social services records (which she had maintained in triplicate form to ensure she would not lose any important documents); successfully engaged her elected representative to advocate for her disability claim; created a household budget; and planned and executed three trial weekends caring for her children without outside help. With these tangible achievements and improvement in self-efficacy, M reported feeling "like a good mother." 


\section{Discussion}

ADHD is a neurodevelopmental disorder characterized by symptoms such as decreased selective attention and concentration, impulsivity, and hyperactivity experienced throughout the lifespan, although the degree of impairment caused by these symptoms might differ according to the situation and/or task at hand. Deficits are more pronounced for tasks that are "boring" for the individual, as well as ones that demand sophisticated executive functioning for prolonged periods of time. The disorder is chronic, but not constant [8]. Without adequate treatment, the individual finds several ways to compensate for these deficits throughout childhood and later in their adult life. In adult women with untreated ADHD, difficulties managing social and professional demands contribute to chronic low self-esteem and decreased self-confidence, thus predisposing them to incapacitating anxiety and depressive symptoms. Some women may resort to illicit substance use (especially cocaine and stimulants) to cope with untreated symptoms, which further predisposes them to trauma and long-term deficits in executive functioning, as well as potential involvement with the legal system and child welfare agencies.

Throughout a woman's lifespan, ADHD symptoms can vary in response to hormonal changes. The earlier brain maturation seen in women as compared to men may be protective, but the increased release of estrogen during puberty and the corresponding increase in dopamine receptors may lead to a spike in symptoms [9]. Clinically, this might present as variation in intensity of ADHD symptoms during the menstrual cycle. Likewise, hormonal fluctuations during pregnancy and the post-partum period may exacerbate these symptoms, increasing the woman's risk of postpartum psychiatric complications and substance use.

Our patient's treatment course highlights treatment complexities and innovative ways of managing these issues. The long-term treatment of challenging cases like this one may seem daunting, especially for early career health professionals. The treatment we describe hinged on creative problem solving with the patient within the context of our therapeutic alliance. While the treatment interventions used and described here are not novel in themselves, our implementation of this integrated approach is unique and highlights the effectiveness of individualized treatment for perinatal women struggling with dual diagnosis. In our literature review of treatment interventions for dual diagnoses, specifically during the perinatal period and child rearing, we were unable to find other case reports detailing symptomology and treatment interventions similar to M's case. This complex presentation, however, is not uncommon in outpatient psychiatric settings, with the main clinical challenge being persistent engagement in treatment and minimizing risk of relapse. Within this author's scope of practice, M's case is one where sobriety has been maintained the longest (3.5 years) with a significant improvement in functioning with treatment of ADHD symptoms. We believe this is secondary to combining pharmacological and psychotherapeutic interventions (attachment-based interventions, ADHD coaching), and maintaining weekly contact for ongoing encouragement, positive reinforcement and helping her complete one task at a time (in M's words, "staying on track").

\section{Treatment challenges and considerations}

Over the course of this patient's treatment, we adjusted our treatment approach to accommodate M's needs at each phase of her recovery and in her evolving role as a mother.

In the initial phase of treatment, engaging this patient was a struggle. Chaos and social stressors posed significant barriers to continuing treatment. Struggles with initial as well as long- term engagement in treatment are inherent in substance use treatment. Untreated ADHD adds to this problem leading to issues such as missed appointments, late arrivals, easy distractibility and poor medication compliance. Furthermore, for mothers with ADHD, guilt and shame over their inability to meet social and maternal expectations may prevent them from seeking the appropriate medical help. Limitations related to deficient executive functioning hinder their ability to navigate through complicated legal systems and child welfare agencies.

Deficits in higher cognitive functioning--including difficulties with voluntary shifts in attention required for toggling between various tasks, an inability to sustain selective focus required to complete a task, and poor sense of time leading to taking on too many tasks (which subsequently is self-defeating) --reinforce the individual's feelings of inadequacy and worthlessness. These challenges in daily functioning can be perplexing when they compare their intellect to their counterparts. Since these deficits are not constant throughout all higher functions, these women might be able to do some tasks very well using their creativity, high energy and a constant inflow of ideas, but might fail to complete tasks that require sophisticated executive function and seem to "come naturally" to their counterparts. Thus, the subjective experience of being a mother with ADHD is often a source of bewilderment and shame.

The heightened social demands of the post-partum role transition are difficult for most women; for mothers with ADHD, it can feel impossible. To cope, these women feel a pressing need for structure. For M, this manifested in obsessive list-making. In her self-described "forgetfulness" and "carelessness" (deficits in working memory and attention), she would make multiple lists on post-it notes to keep track of her daily progress. Adding to her sense of frustration, M would often misplace these papers, subsequently spending more time and energy to create new ones. Therefore, treatment interventions involved use of organizational skills, including creation of a digital to-do list designed to help meet short-term goals. 
Another vital psychological process inherent in the postpartum period is attachment within the mother-baby dyad. The importance of secure attachment in learning to effectively selfregulate through co-regulation in social relationships cannot be emphasized enough. Numerous studies show individuals who develop insecure attachment patterns during early childhood are likely to have long-term difficulties and tumultuous social relationships during adulthood [10]. Synchrony in mother-baby interactions aids in forming secure attachments and ascribing meaning to events (mentalization) [11]. Emotional regulation and judicious reciprocal interactions within the mother-baby dyad are fundamental to this early developmental process [12]. Synchrony requires that the mother gives her curious attention to cues presented by the infant while they express their basic needs. ADHD related deficits in cognition can impair selective and prolonged attention, thus interfering with this process. Events characteristic of this period, like incessant loud crying of the infant; disruptive behavior of older children; multi-tasking, and increased social interactions (other parents, teachers) can produce sensory over-stimulation. This sensory overload, in turn, increases distractibility and predisposes the patient to feel overwhelmed and dysregulated (affectively and behaviorally). In an attempt to cope, the patient might withdraw or become hypervigilant/hyper-responsive to infant cues; either extreme will hinder secure attachment.

M's treatment at our partial hospital program emphasized mindfulness skills which reduced distractibility to both internal and external stimuli, and enhanced metacognition. Emotion regulation skills improved frustration tolerance, concentration for longer periods of time, impulsivity and mood-driven behaviors. We offered an overall corrective emotional experience with the goal of improving M's social attachments within the microcosm of our program (other clinicians and patients), as well as her capacity for reflective functioning in relation to her children. Crucially, these attachments helped her to remain engaged in long-term treatment (currently ongoing).

From a psychopharmacology standpoint, our primary focus was to treat M's ADHD-related symptoms with relapse prevention and maintenance of psychiatric stability. After an unsuccessful trial of non-stimulant medications (poor response and tolerability), we carefully prescribed mixed amphetamine salts with firm structure and close clinical monitoring. Medication management was intentionally formulated to avoid labeling stimulants as the only remedy. Instead, we helped M to prioritize tasks with high efficacy in the hours after taking the medication. Hence, stimulant use only laid the groundwork for optimization of functioning; these skills in the context of "coaching" were essential. We educated M about the effects that chronic mental illness, substance use, and TBIs could have had on her brain. Through empathy and positive reinforcement, we sought to broaden her understanding of her unique brain functions, thus enhancing her strengths, while providing support to cope with weaknesses without guilt or shame.

Lastly, we helped $M$ to meet the demands of her six-year-old son who had significant behavioral and academic problems. M needed strategies to cope not only with her symptoms but her son's ADHD as well. These challenges intensified during the COVID-19 school closures and subsequent distance-learning. With accurate medication management and training in strategic planning (e.g., weekly consultation with her son's teacher, previewing lessons, setting up the computer every night in preparation for the next day's work), M was able to reorganize and provide structure for her son. In therapy, M challenged catastrophic ruminations that her son would be irrevocably disadvantaged by her disabilities and used mindfulness to ground herself in daily practical goals, gaining mastery over one goal at a time.

\section{Conclusion}

The perinatal period and subsequent transition to motherhood presents greater challenges for women with ADHD as compared to neurotypical mothers, particularly for dual diagnosis patients who frequently use substances to selfmedicate distress related to untreated deficits. In M's case, our goal was to build a strong therapeutic alliance, closely monitor clinical symptoms and response to medications, and conduct strengths-based psychotherapy conceptualized as ADHD coaching. These interventions optimized her ability to care for her children and household, resulting in increased self-efficacy as a mother, improved self-regard, and sustained sobriety. Though time consuming and labor intensive, this type of long-term, multi-disciplinary and individualized treatment approach may be replicable in other clinical settings. More clinical research is required in varied clinical settings to study system specific limitations. As demonstrated through M's case, such interventions can improve the quality of life for mothers as well as their children, thus increasing the potential for better long-term outcomes.

\section{References}

1. Kessler RC, Adler L, Barkley R, Demler O, Joseph B, et al. (2006) The prevalence and correlates of adult ADHD in the United States: results from the National Comorbidity Survey Replication. Am J Psychiatry 163: 716-723.

2. Gershon J (2002) A meta-analytic review of gender differences in ADHD. Journal of Attention Disorders 5(3): 143-154.

3. Biederman J, Petty CR, O'Connor KB, Hyder LL, Faraone SV (2012) Predictors of persistence in girls with attention deficit hyperactivity disorder: results from an 11-year controlled follow-up study. Acta Psychiatr Scand 125(2): 147-156.

4. Biederman J, Petty CR, Monuteaux MC, Fried R, Byrne D, et al. (2010) Adult psychiatric outcomes of girls with attention deficit hyperactivity disorder: 11-year follow-up in a longitudinal case-control study. Am J Psychiatry 167(4): 409-417.

5. Cassoff J, Wiebe ST, Gruber R (2012) Sleep patterns and the risk for ADHD: a review. Nature and science of sleep 4: 73-80. 
6. Halperin, JM, Healey, DM (2011) The influences of environmental enrichment, cognitive enhancement, and physical exercise on brain development: can we alter the developmental trajectory of ADHD? Neuroscience and biobehavioral reviews 35(3): 621-634.

7. Freeman MP (2014) ADHD and pregnancy. Am J Psychiatry 171(7): 723-728.

8. Brown TE (1999) New understandings of Attention Deficit Disorders in Children, Adolescents and Adults: Assessment and Treatment. Focus, The official newsletter of the National Attention Deficit Disorder Association.

9. Ostojic D, Miller C (2014) Association between puberty and symptoms of ADHD in female university students. Journal of attention disorders 20(9): 782-791.

10. Stovall-McClough K, Dozier M (2016) Attachment Stated of Mind and Psychopathology in Adulthood. In book: Handbook of attachment theory and research, Edition III. Guilford Press: 715-738.

11. Fonagy P, Cambell C (2017) Mentalizing, attachment and epistemic trust: how psychotherapy can promote resilience. Psychiatr Hung 32(3): 283-287.

12. Leclere C, Viaux S, Avril M, Achard C, Cohen D, et al. (2014) Why synchrony matters during Mother-Child Interactions: A systematic review. PLoS One 9(12): e113571.

\section{Your next submission with Juniper Publishers} will reach you the below assets

- Quality Editorial service

- Swift Peer Review

- Reprints availability

- E-prints Service

- Manuscript Podcast for convenient understanding

- Global attainment for your research

- Manuscript accessibility in different formats ( Pdf, E-pub, Full Text, Audio)

- Unceasing customer service

Track the below URL for one-step submission https://juniperpublishers.com/online-submission.php 